Maria Stofa, Theodoros Xanthos, Konstantinos Ekmektzoglou, Athanasios Chalkias, Dimitrios Barouxis, loannis Pantazopoulos, Nicoletta lacovidou

School of Medicine, University of Athens, Greece

\title{
Quality of life in adults with cystic fibrosis: the Greek experience
}

The authors declare no financial disclosure

\begin{abstract}
Introduction: Recent developments in treatment have steadily raised the median predicted age of survival for people with Cystic Fibrosis (CF). We report the health-related quality of life (HROoL) in CF adult patients and correlate our findings with the patients' demographic characteristics.

Material and methods: The Cystic Fibrosis Quality of Life (CFOoL) questionnaire was answered by 77 CF adult patients. The questionnaire included questions pertaining to age, sex and level of education and covered eight sections of functioning.

Results: The highest score was reported in the "Social Functioning" section, while the lowest in the "Concerns for the Future" section. When different age groups were compared, statistical significances were reported in "Physical Functioning", "Interpersonal Relationships", and the "Career Concerns" section, with older patients reporting statistically higher HROoL scores than younger ones $(p<0.005)$. No statistically significant difference was reported amongst the scoring between male and female CF patients. When different educational levels were compared, patients that had received a higher educational training scored statistically higher in all but one sections of the questionnaire when compared with patients of a lower educational level $(p<0.005)$.

Conclusion: More than half Greek adult CF patients report that they are capable to participate in social activities but most of them are worried about the outcome of their disease and its effect on their lives.
\end{abstract}

Key words: cystic fibrosis, quality of life, adults, Greece

Pneumonol Alergol Pol 2016; 84: 205-211

\section{Introduction}

CF, the commonest severe autosomal recessive disorder in Caucasians with an average rate of 1/1,800-8,000 living births, affects approximately 80,000 patients worldwide [1-3]. Although recent developments in treatment have steadily raised the median predicted age of survival for people with CF over the last 25 years (in 2011, the age was 36.8 years) [4], the topic of health-related quality of life (HRQoL) measurement in CF remains still under intense scrutiny and research.

Standard guidelines of CF care include a multidisciplinary approach to the patient with CF (nutrition, lung health, infection control, age -specific care) [3]. However, an integral part of the patient's well being is the HRQoL, which must evaluate any physical symptom, the patient's functional status, their psychological and emotional state as well as any level of social functioning [5]. The patient's perspective (as depicted through the HRQoL) can be an extremely helpful as it can guide both research and clinical practice [6].

In this institutional study, we report, for the first time in Greece, the HRQoL in CF adult patients with the use of a questionnaire and correlate our findings with the patients' age, gender and educational level.

\section{Material and methods}

\section{Questionnaire}

The Cystic Fibrosis Quality of Life (CFQoL) questionnaire was used, as previously described [7]. We chose this appropriately validated for use in CF studies questionnaire since it has not only

Address for correspondence: Konstantinos Ekmektzoglou, School of Medicine, University of Athens, Greece, e-mail: ekmektzo@hotmail.com DOI: 10.5603/PiAP2016.0025

Received: 13.12 .2015

Copyright (C) 2016 PTChP

ISSN 0867-7077 
Table 1. The sections covered by the questionnaire

\begin{tabular}{lccc}
\hline Section & $\begin{array}{c}\text { Number } \\
\text { of questions } \\
(\mathbf{5 2 )}\end{array}$ & Score from $\mathbf{1}$ to $\mathbf{6}$ & $\begin{array}{c}\text { Cronbach's } \\
\text { Alpha }\end{array}$ \\
\hline Physical functioning & 10 & All the time/most of the time/good bit of the time/sometimes/occasionally/never & 0.959 \\
Social functioning & 4 & All the time/most of the time/good bit of the time/sometimes/occasionally/never & 0.892 \\
Treatment & 7 & All the time/most of the time/good bit of the time/sometimes/occasionally/never & 0.819 \\
Treatment issues & 4 & & 0.910 \\
Chest symptoms & 3 & All the time/most of the time/good bit of the time/sometimes/occasionally/never & 0.941 \\
Emotional functioning & 8 & Strongly agree/agree/slightly agree/slightly disagree/disagree/strongly disagree & 0.845 \\
Concerns for the future & 6 & Strongly agree/agree/slightly agree/slightly disagree/disagree/strongly disagree & 0.941 \\
Interpersonal & 10 & & \\
relationships & 3 & Strongly agree/agree/slightly agree/slightly disagree/disagree/strongly disagree & 0.832 \\
Body image & 4 & Strongly agree/agree/slightly agree/slightly disagree/disagree/strongly disagree & 0.959 \\
Career concerns & & &
\end{tabular}

been previously used on an adult CF population but has, also, undergone appropriate psychometric testing in $\mathrm{CF}$ [6].

The questionnaire included questions pertaining to age, sex and level of education $(n=3)$ and covered eight sections of functioning $(n=52)$. The scoring scales were designed to reflect positive and negative scoring (Table 1).

\section{Participants and data collection}

After explanation of the purposes of the study, $77 \mathrm{CF}$ patients agreed to respond to the CFQoL questionnaire. The diagnosis of $\mathrm{CF}$ consisted of finding specific clinical (phenotypic) characteristics in combination with biochemical or genetic markers of CF transmembrane conductance regulator (CFTR) dysfunction, as proposed by the European Union Cystic Fibrosis Diagnostic Working Group [8]. The patients were assured that participation was voluntary and that they were free to withdraw any time they wanted. Their anonymity in completing the questionnaire was also assured along with the remark that any data collected would not be used for other purposes. No participant withdrew from the survey. Data collection was performed from January $1^{\text {st }} 2013$ to June $1^{\text {st }} 2013$ by the same investigator. All data were saved in a database. The survey was performed in the adult Cystic Fibrosis Unit of the "Sismanogleio" General Hospital of Attica, Greece.

No patient had undergone lung transplantation in the past.

\section{Statistical analysis}

The score of all the questions was added. All items were scored 1 to 6 (except question 6 of the
"Physical Function" Section which had a reverse scoring). Raw scores of 3 or under indicated a negative response. Transformed scores that translated to values between 0 and 100 indicated the value that had been achieved out of a maximum of 100 with 100 indicating the most positive HRQoL levels possible. The Cronbach's Alpha factor was calculated so as to check the reliability of the aforementioned scores. In order to compare the HRQoL with the basic demographic data, a one way fluctuation analysis was used (ANOVA F-test), the middle value was compared with 2 independent samples (t-test) and the Spearman $\mathrm{r}$ correlation coefficient was calculated.

A p-value $<0.05$ was considered statistically significant. Data were analyzed using the statistical software for social sciences (SPSS, Chicago, USA, version 17).

\section{Results}

Response ratio was $100 \%$. No patient refused to participate in the study. The demographic characteristics of the study population are shown in Table 2. The Cronbach's Alpha factor for the sum of the scores was above $80 \%$ (minimum $81.9 \%$ and maximum 95.9\%), revealing remarkable reliability in our results (Table 1).

The highest score $(62.5 / 100)$ was reported in the "Social Functioning" section, while the lowest (26.6/100) in the "Concerns for the Future" section (Fig. 1). Patients reported a lower HRQoL in the sections of "Body Image", "Interpersonal Relations" and "Carrer Concerns".

When the HRQoL was compared to the patients' gender, there was no statistically signi- 
ficant difference amongst the scoring reported between male and female CF patients ( $\mathrm{p}>0.05)$. However, the questionnaire revealed some score differences indicative of some tendencies. In the "Treatment Issues" subsection, the middle score difference between female and male patients was 7.1 indicating that male patients have poorer HRQoL ( $p=0.291)$. Likewise, in the "Concerns for the Future" section, the male patients outscored the female patients by a middle value of $7.4(\mathrm{p}=$

Table 2. Demographic characteristics of the study population

\begin{tabular}{ccc}
\hline & Patients (n = 77) & (\%) \\
\hline Gender & & \\
Male & 34 & 44.2 \\
Female & 43 & 55.8 \\
Age (years) & & \\
$18-23$ & 22 & 28.7 \\
$24-28$ & 18 & 23.3 \\
$29-33$ & 18 & 22.3 \\
$>33$ & 19 & 24.7 \\
Educational level & & \\
None & 4 & 5.2 \\
Primary & 6 & 7.8 \\
Secondary $^{2}$ & 26 & 33.8 \\
Tertiary $^{3}$ & 41 & 53.2 \\
\hline
\end{tabular}

'Primary education pertains to primary school spanning six years (ages 6 to 12) ${ }^{2}$ Secondary education comprises two stages: a compulsory three-year school (middle or junior high school), followed by a non compulsory three-year academically oriented high school (unified upper secondary schools or technical-vocational educational institutes (ages 12-18)

${ }^{3}$ Tertiary education is provided by Universities, Polytechnics, Technological Educational Institutes and Academies (ages 18 and above)
0.211). Additionally, in the "Body Image" section, male patients reported lower scores than female patients (middle score difference of 12.2) $(\mathrm{p}=$ 0.118) (Table 3).

When different age groups were compared, in the "Physical Functioning" section, older patients reported statistically higher HRQoL scores than younger ones $(p=0.006)$, with the middle scoring difference between patients 33 years or older and younger patients ranging from 13.7 to 28.5. In the "Interpersonal Relationships" section, patients younger than 33 years reported statistically significant poorer HRQoL by scoring less in the questionnaire $(p=0.039)$, with the middle scoring difference between patients 33 years or older and younger patients ranging from 10.2 to 25 . Besides, the analysis revealed a statistical deference in the "Career Concerns" section, since older patients scored higher than younger ones. The middle score difference in the 33 years or older group when compared to the others ranged from 5.7 to 30.8 ( $\mathrm{p}=0.013$ ) (Table 3 ).

Finally, when analyzing the patients' educational level, in the "Physical Functioning" section, patients who had received tertiary educational training reported statistically higher scores when compared to other patients. The middle score difference was 41.5 in comparison to patients who had received none/primary educational training and 18 to those with secondary educational training $(p<0.001)$. Similar results were reported in the "Social Functioning" section where the middle score difference was 31.2 and 15.2 , respectively

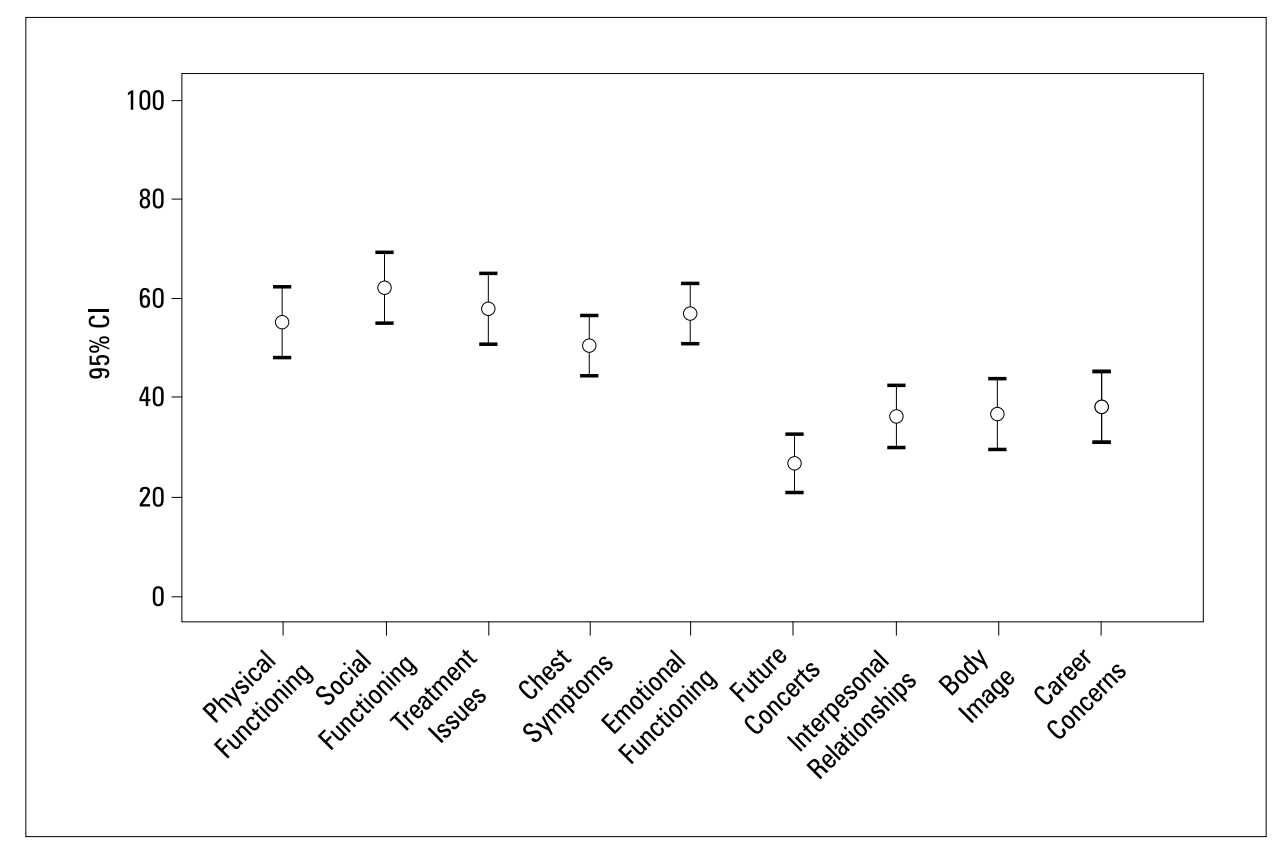

Figure 1. The graph depicting the middle value and the $95 \% \mathrm{Cl}$ for the section scores in patients with cystic fibrosis 


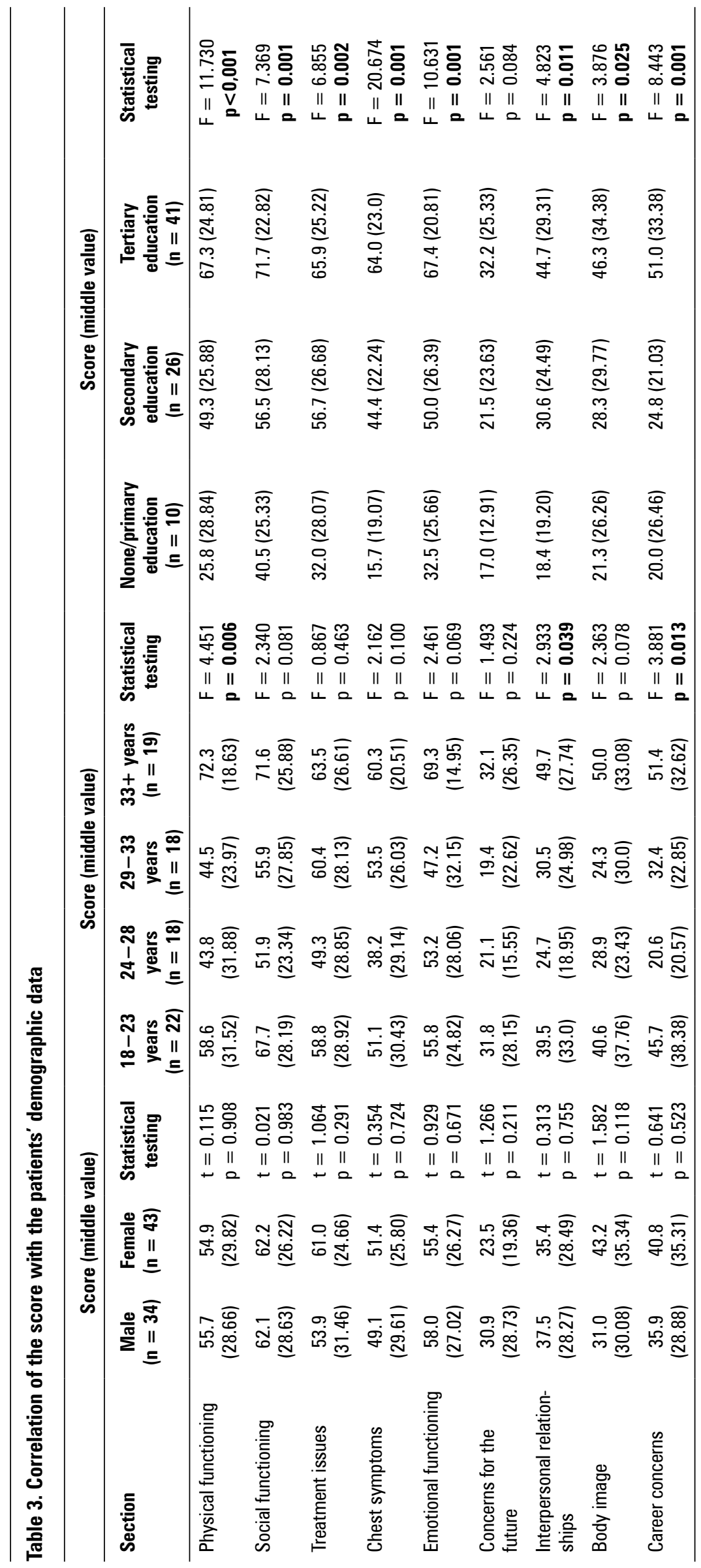


$(\mathrm{p}=0.001)$. Patients who had received none/primary and secondary educational training scored significantly lower when compared to tertiary education patients in the "Treatment" section. More specifically, the middle score difference was 33.9 and 9.2, respectively, in the "Treatment Issues" subsection $(p=0.002)$ and 48.3 and 19.6, respectively, in the "Chest Symptoms" subsection (p $=0.001$ ). Furthermore, patients with lower educational training reported significantly lower scores as opposed to tertiary educational level patients in the "Emotional Functioning" section (middle score difference was 34.9 for the none/primary educational level patients and 17.4 for the secondary educational level patients when compared to the tertiary educational level group, $\mathrm{p}=0.001$ ). Regarding the "Interpersonal Relationships" section, patients with a tertiary education training reported a 26.3 score difference when compared to patients with no/primary education and 13.4 score difference when compared to patients with secondary education $(\mathrm{p}=0.011)$. In the "Body Image" section, results were similar with patients having received a tertiary education training reporting a statistically higher HRQoL (middle score difference of 25 with no/primary educational level patients and 18 with secondary educational level patients) ( $p=0.025)$. In the "Career Concerns" section the scoring revealed that patients of a lower educational level reported a statistically lower HRQoL, with the middle score difference between patients of tertiary educational level and patients of no/primary educational level being 31 and that between patients of tertiary educational level and patients of secondary educational level being 26.2 ( $p=0.001)$. Interestingly enough, although not statistically significant, the results revealed higher scores reported by patients having received a tertiary education than patients of a lower educational level in the "Concerns for the Future" section (middle score difference of 15.2 and 10.7, respectively) $(\mathrm{p}=0.084)$.

\section{Discussion}

The definition of HRQoL comprises of several multifactorial domains as reported by the patient $\mathrm{him} /$ herself. It is of the outmost importance in clinical practice as the correlations between clinical variables and HRQoL are often poor. It is the HRQoL that can provide valuable information not reported by other outcomes. The patients' perspective regarding their well being or how they cope with the treatment can provide a reliable and cheap way of standardizing and evaluating therapeutic schemes and interventions [9]. Therefore, HRQoL scales can be used (except from presenting the illness impact on the patients' daily functioning and coping) to guide clinical decision, to estimate the cost effectiveness of a specific drug, to evaluate surgical interventions, and, last but not least, to report outcomes in clinical trials $[10,11]$.

Since, as described above, the HRQoL is, by definition, a multidimensional concept, it is logical that different types of scales have been used in CF studies with, sometimes, inconsistent findings. Scientists and researchers agree that there is no "gold standard" on measuring HRQoL in CF patients through the use of questionnaires. Furthermore, the disease itself has had many breakthroughs (regarding available therapeutic modalities) since the first attempts to measure HRQoL were undertaken, rendering the significance of previous reported data sometimes questionable [6]. Our study is the first study in Greece that reports the HRQoL in CF adult patients and associates these results with their demographic characteristics.

Regarding the HRQoL, the highest score was reported in the "Social Functioning" section and the lowest in the "Concerns for the Future" section, rendering more than half of these patients capable to participate in social activities. However, most of them are worried about the outcome of their disease and its effect on their lives. Besides, the consistent low scores reported on the sections of "Body Image", "Interpersonal Relations" and "Career Concerns" clearly indicate that Greek adult CF patients are characterized by low self esteem that reflects on how they react and cope with others and are very skeptics regarding any chance for professional evolvement.

Studies have reported that CF patients of increased age score less in respect of physical and social functioning, career concerns and interpersonal relationships when measuring their HRQoL. On the other hand, they seem to report a better HRQoL when measuring their concerns for the future, perhaps due to their constant re-evaluation through time of what really matters in life $[12,13]$. Our study revealed that when different age groups were compared, older patients (33 years and older) scored less both in the "Physical Functioning" as well as in the "Interpersonal Relationships" section, as expected. However, they also reported a better HRQoL regarding their "Career Concerns", in contrast with other findings. Perhaps the better clinical outcomes reported by the advantages in CF therapy makes 
our study group cope better regarding its career expectations. No other statistical significance was noted in our study.

Clinical studies in CF populations have, also, confirmed that there are gender differences in the reported HRQoL with women of any age (children, adolescents, and adults) scoring less in HRQoL scales than men [14-16]. Female patients have reported a poorer HRQoL when it comes to physical, emotional and psychological functioning as well as chest symptoms (even girls have reported poorer chest symptoms than boys with no difference in their FEV 1 ) [6, 17]. Interestingly enough, women seem to have a more accurate perception of their objective clinical status than men [16]. This might explain the fact that in some studies women have scored more in the HRQoL questionnaire regarding interpersonal relationships and body image [12, 13, 18, 19]. However, the aforementioned results could not be validated in our study since we showed no statistical significance between adult gender groups. Nevertheless, our data revealed a trend of women scoring less in the "Treatment Issues", the "Concerns for the Future" and the "Body Image" section. Perhaps the small number of patients (when compared to other published studies) or the fact that no clinical (i.e. $\mathrm{FEV}_{1}$ ) or psychosocial variables (i.e. depression, anxiety) were taken into account could explain these discrepancies in results.

When trying to compare our results with other studies, data are interesting. A recent study investigating factors related to changes in the quality of life among Polish adolescents and adults with CF over a 1-year period, revealed that better baseline spirometry (and, therefore, systematic chronic therapy) was a predictive factor for improvement in treatment issues and career concerns. Maintaining good physical condition and activity seemed to correlate positively with future and career concerns, while residents in rural areas or living under difficult conditions were especially vulnerable to deterioration in future concerns [20].

The American Project on Adult Care in CF, a prospective, longitudinal panel study of 333 adult CF patients, assessed both physical and psychosocial domains of health via the CF Questionnaire-Revised (CFQ-R), seven times over 21 months. The results revealed that lung function, frequency of pulmonary exacerbations, and nutritional indices were associated with physical CFQ-R domain scores, as expected. However, population changes in several psychosocial domains of the CFQ-R suggested that differentiating between the physical and the psychosocial trajectories in health among adults with CF is critical in evaluating patient-reported outcomes [21].

To make things even more difficult for the physician to interpret, only recently, Abbott et al. undertook a longitudinal observational study enrooling 234 participants aged 14-48 years from large adult CF centres in the United Kingdom. Observations were obtained at seven time points: approximately every 2 years over a 12 -year period. The authors revealed that demographic and changes in clinical variables were independently associated with a change in HRQoL over time. They came to the conclusion that cross-sectional data are inadequate when evaluating the relationships between HRQoL domains and key demographic and clinical variables, as they fail to recognise the full impact of the CF disease trajectory and its treatments on quality of life [22].

Our study is the first one to report the influence of the patients' educational level on their HRQoL. Patients that had received a higher (tertiary) educational training scored statistically higher on almost all sections of the questionnaire when compared with patients of a lower educational level. Even in the "Concerns for the Future" section, the data analysis revealed a tendency for higher scores amongst better educated patients. Given the fact that the patients' educational training should lead to a more fulfilling job and that the increased life expectancy in CF patients has helped more people with $\mathrm{CF}$ entering the workforce [23], it is possible for the work environment to provide a form of social scaffolding and make better educational trained $\mathrm{CF}$ individuals cope better with their disease. A recent HRQoL evaluation in a Polish adolescent population with CF (aged 14-18 years) revealed that school attendance had a significant impact on many aspects of the adolescents' functioning [24], validating, in part, our findings.

\section{Limitations}

As mentioned before, the number of patients was relative small when compared to other studies. Besides, no clinical or psychosocial variables were taken into consideration when analyzing the patients' reported HRQoL. As, already reported, the predicted $\mathrm{FEV}_{1} \%$ and pulmonary exacerbations have the broadest impact on HRQoL in CF patients [19].

Last, but not least, our study did not assess the financial status of each patient and its effect on their quality of life. The BURQOL-RD Research Network investigating the social economic bur- 
den and health-related quality of life in patients with rare diseases in Europe, has shown that the annual cost for a CF patient is high, with indirect costs relating to informal care and early retirement baring significant societal implications. Studies so far, have revealed that in economic vulnerable populations the risk factors for worse health outcomes are increased [25, 26]. This could be of paramount importance for a country like Greece, facing over the last years a dramatic economic crisis with tremendous implications in the well-being of each patient.

\section{Conclusions}

Repeated HRQoL measurement through clinical studies should be undertaken so as to improve the care delivered to patients and provide useful information concerning the individual's adaptation to the disease. Patients' outcomes should be reported in well planned clinical trials as an additional outcome parameter. Our results showed that a higher educational training seems to improve the quality of life of adult CF patient; perhaps a better and more rewarding working environment can enhance the patients' ability to cope with their disease.

\section{Conflict of interest}

The authors declare no conflict of interest.

\section{References:}

1. Farrell PM. The prevalence of cystic fibrosis in the European Union. J Cyst Fibros 2008; 7: 450-453.

2. Rowe SM, Borowitz DS, Burns JL et al. Progress in cystic fibrosis and the CF Therapeutics Development Network. Thorax 2012; 67: 882-890.

3. O'Sullivan BP, Freedman SD. Cystic fibrosis. Lancet 2009; 373: 1891-904.

4. 2011 annual data report to the center directors. Bethesda, MD Cystic Fibrosis Foundation Patient Registry, 2012; URL:www. cff.org/UploadedFiles/research/ClinicalResearch/2011-Patient -Registry.pdf; 14.06.2014.

5. Spilker B. Introduction. In: Spilker B (ed.) Quality of life and pharmacoeconomics in clinical trials. Lippincott-Raven, Philadelphia 1996; 1-10.

6. Abbott J. Health-related quality of life measurement in cystic fibrosis: advances and limitations. Chron Respir Dis 2009; 6: 31-41.

7. Gee L, Abbott J, Conway S, Etherington C, Webb, AK. Development of a disease specific health related quality of life measure for adults and adolescents with cystic fibrosis. Thorax 2000; 55: $946-954$
8. De Boeck K, Wilschanski M, Castellani C et al. Cystic fibrosis: terminology and diagnostic algorithms. Thorax 2006; 61: $627-635$.

9. Goldbeck L, Schmitz TG. Comparison of three generic questionnaires measuring quality of life in adolescents and adults with cystic fibrosis: the 36-item short form health survey, the quality of life profile for chronic diseases, and the questions on life satisfaction. Qual Life Res 2001; 10: 23-36.

10. Gold MR, Franks P, McCoy KI, Fryback DG. Toward consistency in cost-utility analyses: using national measures to create condition-specific values. Med Care 1998; 36: 775-777.

11. Kaplan RM. Health outcome models for policy analysis. Health Psychol 1989; 8: 723-735.

12. Gee L, Abbott J, Hart A, Conway SP, Etherington C, Webb AK. Associations between clinical variables and quality of life in adults with cystic fibrosis. J Cyst Fibros 2005; 4: 59-66.

13. Schmitz TG, Henrich G, Goldbeck L. Quality of life with cystic fibrosis - aspects of age and gender. Klin Padiatr 2006; 218: $7-12$.

14. Modi AC, Quittner AL. Validation of a disease specific measure of health related quality of life for children with cystic fibrosis. J Pediatr Psychol 2003; 28: 535-546.

15. Arrington-Sanders R, Yi MS, Tsevat J, Wilmott RW, Mrus JM, Britto MT. Gender differences in health-related quality of life of adolescents with cystic fibrosis. Health Qual Life Outcomes 2006; 4: 5 .

16. Gee L, Abbott J, Conway SP, Etherington C, Webb AK. Quality of life in cystic fibrosis: the impact of gender, general health perceptions and disease severity. J Cyst Fibros 2003; 2: $206-213$.

17. Sawicki GS, Sellers DE, Robinson WM. Self-reported physical and psychological symptom burden in adults with cystic fibrosis. J Pain Symptom Manage 2008; 35: 372-380.

18. Abbott J, Morton AM, Musson $\mathrm{H}$ et al. Nutritional status, perceived body image and eating behaviours in adults with cystic fibrosis. Clin Nutr 2007; 26: 91-99.

19. Habib AR, Manji J, Wilcox PG, Javer AR, Buxton JA, Quon BS. A systematic review of factors associated with health-related quality of life in adolescents and adults with cystic fibrosis. Ann Am Thorac Soc 2015; 12: 420-428.

20. Dębska G, Mazurek H Factors related to changes in the quality of life among Polish adolescents and adults with cystic fibrosis over a 1-year period. Patient Prefer Adherence 2015; 9: $1763-1770$.

21. Dill EJ, Dawson R, Sellers DE, Robinson WM, Sawicki GS. Longitudinal trends in health-related quality of life in adults with cystic fibrosis. Chest 2013; 144: 981-989.

22. Abbott J, Morton AM, Hurley MA, Conway SP. Longitudinal impact of demographic and clinical variables on health-related quality of life in cystic fibrosis. BMJ Open 2015; 5: e007418.

23. Goldbeck L, Zerrer S, Schmitz TG. Monitoring quality of life in outpatients with cystic fibrosis: feasibility and longitudinal results. J Cyst Fibros 2007; 6: 171-178.

24. Borawska-Kowalczyk U, Sands D. Determinants of health-related quality of life in polish patients with CF - adolescents' and parents' perspectives. Dev Period Med 2015; 19: 127-136.

25. Iskrov GG, Stefanov RS, López-Bastida J et al. Economic burden and health-related quality of life of patients with cystic fibrosis in Bulgaria. Folia Med (Plovdiv) 2015; 57: 56-64.

26. Angelis A, Kanavos P, López-Bastida J et al. Social and economic costs and health-related quality of life in non-institutionalised patients with cystic fibrosis in the United Kingdom. BMC Health Serv Res 2015; 15: 428. 